\section{Adopción de estilos de vida como estrategia en la prevención y control de las enfermedades no transmisibles}

\author{
Olga Marina Vega Angarita*
}

En los últimos decenios, los cambios en los patrones demográficos y epidemiológicos de la población de muchos países aumentaron la relevancia relativa de las enfermedades no transmisibles (ENT) y sus factores de riesgo como condicionantes en su prevalencia. Entre los determinantes de las ECNT, existen un conjunto de factores de riesgo comportamentales que están relacionados con modos de vida susceptibles de modificación (1).

Por naturaleza las ENT son incurables, su progresión genera mayores complicaciones en la salud y depende de la fisiopatología, la eliminación de hábitos perjudiciales y la adopción de pautas que generen control y estabilidad. Se caracterizan por su carácter complejo y por depender de factores genéticos, fisiológicos, ambientales y conductuales de la persona. Estos últimos son prevenibles y controlables (2).

Conviene subrayar, que la adopción de estilos de vida saludables como alternativa en la atención de las enfermedades no transmisibles (ENT) cobra especial relevancia al ser un reto que enfrentan los sistemas de salud dada la incidencia cada vez más notoria a la morbilidad, la mortalidad y la discapacidad. También influyen en la carga económica que representan los servicios de salud, pues el $80 \%$ de los gastos de atención médica son absorbidos en el manejo de estas enfermedades (3).

De esto dan cuenta Serra et al. (4), quienes citando a la Organización Mundial de la Salud (5) refieren que son responsables del fallecimiento las enfermedades cardiovasculares (17,7 millones cada año), seguidas del cáncer (8,8 millones), las enfermedades respiratorias ( 3,9 millones) y la diabetes (1,6 millones). Estos cuatro grupos de enfermedades son responsables de más del $80 \%$ de todas las muertes prematuras por ENT. Todas ellas asociadas a cuatro comportamientos de riesgo modificables: consumo de tabaco, sedentarismo, alimentación poco saludable y consumo excesivo de alcohol (6).

Hay que mencionar, que además al estar bien establecida la función de los comportamientos de riesgo modificables como factores determinantes de enfermedades no transmisibles, los convierte en componentes fundamentales de las actividades de prevención (5). Cada vez más las investigaciones apuntan a la importancia de la adop- ción de estilos de vida y las decisiones de las personas en el mejoramiento de su salud y gestión de las enfermedades no transmisibles $(2,7,8)$.

Se debe agregar, que existen cuatro causas fundamentales como exceso de peso, dieta inadecuada, inactividad física y tabaquismo que son responsables de la mayoría de los casos de mortalidad y morbilidad de la sociedad moderna como: cardiopatías, ictus, cáncer colorrectal, depresión, nefropatías, diabetes, artrosis, osteoporosis y enfermedades neurodegenerativas (9).

En el informe sobre Indicadores Básicos de 2019 "Tendencias de la salud en las Américas", la Organización Panamericana de la Salud (2019) se señala que el sobrepeso y la obesidad (definida como un índice de masa corporal $\geq 25 \mathrm{~kg} / \mathrm{m} 2$ ) son los principales factores de riesgo de aparición de las enfermedades cardiovasculares, la diabetes y algunos tipos de cáncer. La prevalencia del sobrepeso y la obesidad ha aumentado extraordinariamente durante los últimos 20 años. Según algunos datos se estima que un $70 \%$ de la población en los países desarrollados no realiza la suficiente actividad física como para mantener la salud y controlar el peso corporal (10). Por tanto, el ejercicio físico es una práctica que mejora la salud y ejerce un efecto preventivo de las enfermedades no transmisibles.

De acuerdo con la Organización Mundial de la Salud, el estilo de vida se representa en la interacción entre las condiciones de vida y los patrones individuales de conducta, los cuales son determinados por la cultura, la costumbre, y el ambiente en el que se desarrolla la persona. Son definidos por García et al. (11) como patrones de acción multidimensional mantenidos en el tiempo por factores internos y externos (12).

De otra parte, en la sociología de la salud el estilo de vida es un constructo que relaciona la personalidad y los patrones motivacionales con conductas de riesgo o de protección (13). Así pues, el concepto tiene en cuenta tanto aquellos comportamientos que implican un riesgo para la salud como aquellos otros que la protegen.

Se debe agregar que, el estilo de vida, comprende actitudes y valores que se expresan en el comportamiento del

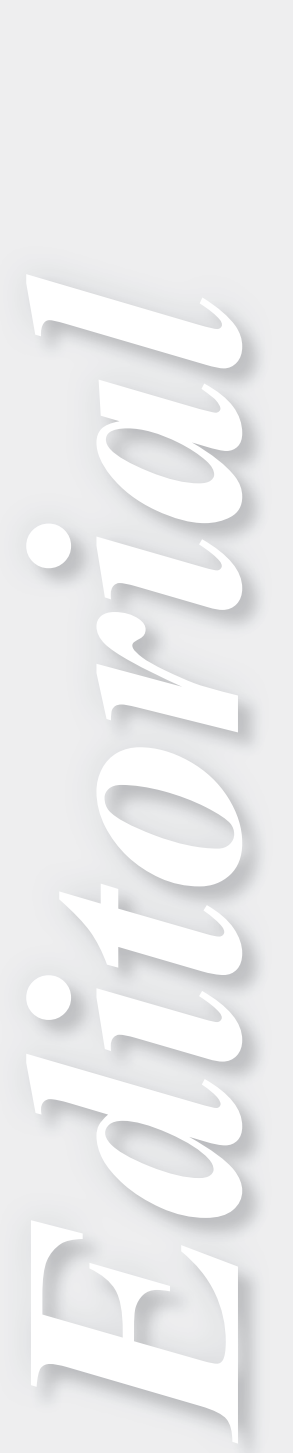

Autor de correspondencia

* Doctora en Educación. Magister Enfermería Cuidado al paciente crónico. Profesora Titular. Programa de Enfermería. Universidad Francisco de Paula SantanderCúcuta. Correo: olgavega@ufps.edu. co Orcid: https://orcid.org/0000-00025525-0088. Cúcuta, Colombia. 
individuo en distintas áreas de la vida, que son influenciadas por los factores socioculturales y características personales. Además de relacionarse con la prevención y control de las principales ENT, tienen que ver con la adopción de pautas que promueven el incremento de conocimientos, cambios de comportamiento y creación de ambientes de apoyo a la adecuada realización de buenas prácticas de salud (13).

La necesidad de comprender la relevancia de los estilos de vida como determinantes de la ENT, así como de intervenirlos con urgencia es una tarea cada vez más importante, pues se reconoce que las acciones del cuidado propio son aprendidas y desarrolladas por las personas en ambientes tanto estables como cambiantes, dentro del contexto de sus patrones de la vida diaria, que son altamente influenciables con acciones (14).

La relevancia de intervenir con medidas preventivas en los diferentes subgrupos poblacionales es posible en cualquier momento y es transferible en los distintos campos de actuación profesional. De ahí, que sea aplicable en: la prevención primaria impidiendo que se desarrolle la enfermedad mediante la modificación de conductas o estilos de vida; la prevención secundaria, mediante la modificación de factores de riesgo para evitar que aparezca la enfermedad; y la prevención terciaria, con la rehabilitación tras un estado patológico y prevención de las recurrencias (15). Son, por tanto. intervenciones que demuestran que la prevención puede ser rentable.

Parte de la gestión profesional de enfermería se centra en la educación de hábitos saludables en las diversas etapas y ámbitos de vida de la persona, desde la promoción hasta el restablecimiento de la salud. Según Achury:

La educación se ha convertido en una herramienta fundamental en la atención integral que involucra a todos los profesionales de la salud, en especial, a los de enfermería, como líderes en el proceso de formación y cambios de conducta de las personas, no solo desde la perspectiva de la prevención y promoción, sino también en el ámbito hospitalario y domiciliario (16).

En definitiva, es un compromiso y una responsabilidad para los profesionales de la enfermería la práctica del cuidado; de ahí, que se deba ofrecerles conocimientos relacionados con la nueva labor, cualificarlos para la prestación de cuidado en situaciones concretas de la enfermedad y fortalecer su papel activo frente a la adopción de estilos de vida adecuados. Igualmente, hay que garantizarles el apoyo institucional y el social, pues representan una alternativa razonable en beneficio de las personas con enfermedad no transmisible.

\section{Referencias}

1. Organización Panamericana de la Salud. Indicadores de salud. Aspectos conceptuales y operativos. [Internet]. Washington: OPS; 2018 [consultado 29 de octubre de 2019]. Disponible en: http://www. deis.msal.gov.ar/wp-content/uploads/2018/07/Indicadores-de-Salud spa.pdf

2. Quillas R, Vásquez C, Cuba M. Promoción de cambios de comportamiento hacia estilos de vida saludable en la consulta ambulatoria. Acta méd. Perú [Internet]. 2017 Abr [citado 2019 Oct 13]; 34(2): 126-131. Disponible en: http://www.scielo.org.pe/scielo.php?script=sci arttext\&pid=S172859172017000200008\&lng=es

3. De La Cruz J, Dyzinger W, Herzog S, Dos Santos F, Villegas F, Ezinga M. Medicina del Estilo de Vida: Trabajando juntos para revertir la epidemia de las enfermedades crónicas en Latinoamérica. Cienc innov salud. 2017; 4(2):1-7.

4. Serra M, Serra M, Viera M. Las enfermedades crónicas no transmisibles: magnitud actual y tendencias futuras. Rev. Finlay [Internet]. 2018 Jun [citado 2019 Oct 13]; 8(2): 140-148. Disponible en: http:// scielo.sld.cu/scielo.php?script $=$ sci arttext\&pid $=$ S2221-24342018000200008\&lng $=$ es

5. Organización Mundial de la Salud. Enfermedades no transmisibles. Nota Descriptiva. [Internet]. Washington: OMS; 2017 [citado 23 Ene 2018].Disponible en: https://www.who.int/es/news-room/ fact-sheets/detail/noncommunicable-diseases

6. Organización Mundial de la Salud. Plan de acción mundial para la prevención y el control de las enfermedades no transmisibles 2013-2020. [Internet]. Washington: OPS; 2013 [consultado 30 de octubre 
de 2019]. Disponible en: https://www.who.int/cardiovascular diseases/15032013 updated revised draft_action_plan_spanish.pdf

7. Vásquez A, Horta L. Enfermedad crónica no transmisible y calidad de vida. Revisión narrativa. Rev. Fac. Cienc. Salud Univ. Cauca [Internet]. 2018 [citado 13 de octubre de 2019]; 20(1):33-40. Disponible en: https://revistas.unicauca.edu.co/index.php/rfcs/article/view/944

8. Villaquiran A, Jácome S, Benavides E. Factores comportamentales para enfermedades no transmisibles en estudiantes Universitarios. Rev. cienc. cuidad. [Internet]. 2018 [citado 13 de octubre de 2019]; 15(2):52-64. https://doi.org/10.22463/17949831.1401

9. De Courten M, De Courten E, Garry Ml. Eidemiologia de la Enfermedad crónica. En Egger Garry Binns Andrew, Rossner Stephan. Medicina del Estilo de Vida. 3.a Edición. Barcelona, España: Elsevier; 2017.

10. Rosa S. Actividad física y salud. Madrid: Díaz de Santos; 2013.

11. García D, García G, Tapiero, Y, Ramos C. Determinantes de los estilos de vida y su implicación en la salud de jóvenes universitarios. (Spanish). Hacia La Promoción De La Salud. 2012; 17(2), 169-185

12. Suescún S, Sandoval C, Hernández F, Araque I, Fagua L, et al. Estilos de vida en estudiantes de una universidad de Boyacá, Colombia. Rev. Fac. Med. 2017; 65 (2): 227-231.

13. García J. Enfoque gerencial de la promoción de la salud. 1a edición. Bogotá: Universidad Nacional de Colombia; 2013.

14. Sabogal I, Mejia C, Plata E, Noriega A, Quintero D, Grimaldo M. Proceso de Enfermería en la persona con diabetes mellitus desde la perspectiva del autocuidado. Rev Cub Enferm. 2017; 33(2): 1.

15. Pérez G, De Juanas O. Calidad de vida en personas adultas y mayores. Edición digital. Madrid: Universidad Nacional de Educación a Distancia; 2013

16. Achury S. Educación para las personas con enfermedad crónica. En Achury DM. Cuidado al paciente con enfermedad crónica. Bogotá: Editorial Pontificia Universidad Javeriana; 2010. 